\title{
Hemoglobin Alc and Angiographic Severity with Coronary Artery Disease: A Cross-Sectional Study
}

\author{
Yugang Yan ${ }^{1,2}$, Ronghua Gao ${ }^{3}$, Shaohui Zhang $\mathbb{D}^{3}$, Zhencai Gao ${ }^{3}$, Anyong Chen ${ }^{3}$, Jianjun Wang ${ }^{3}$, \\ Shufang Zhang ${ }^{3}$, Wen Dai ${ }^{3}$, Fen $\mathrm{Li}^{4}$, Xiangting $\mathrm{Li}^{3}$, Guoliang Yang ${ }^{3}$, Lixin $\mathrm{Liu}^{3}$, Xueying Chen $\mathbb{D}^{2,3}$ \\ 'School of Medical Engineering, Jining Medical University, Jining, Shandong, People's Republic of China; ${ }^{2}$ The Key Laboratory of Cardiovascular \\ Remodeling and Function Research, Chinese Ministry of Education and Chinese Ministry of Health, and The State and Shandong Province Joint Key \\ Laboratory of Translational Cardiovascular Medicine, Qilu Hospital of Shandong University, Jinan, Shandong, People's Republic of China; ${ }^{3}$ Department \\ of Cardiology, Affiliated Hospital of Jining Medical University, Jining, Shandong, People's Republic of China; ${ }^{4}$ Electrocardiogram Room, Jining First \\ People's Hospital, Jining, Shandong, People's Republic of China
}

Correspondence: Xueying Chen, Department of Cardiology, Affiliated Hospital of Jining Medical University, No. 89 Guhuai Road, Jining, 272029, Shandong, People's Republic of China, Tel +86- 537- 2903508, Fax+86 537-2213030, Email chxy22I@I63.com

Background: Many studies have shown that glycated hemoglobin (HbA1c) is associated with coronary artery disease (CAD). HbA1c was independently related to angiographic severity in Chinese patients with CAD after adjusting for other covariates. Some traditional cardiovascular drugs may have an impact on this relationship.

Methods: This retrospective study enrolled a total of 572 CAD patients who underwent their coronary angiography and had their HbA1c levels measured at the Chinese Hospital. The complexity of the coronary artery lesions was evaluated using the Syntax score, and the subjects were divided into 4 inter quartiles according to HbAlc levels. Covariates included history of traditional cardiovascular drugs.

Results: The average age of selected participants was $61.00 \pm 9.15$ years old, and about $54.72 \%$ of them were male. Result of fully adjusted linear regression showed that $\mathrm{HbA1c}$ was positively associated with Syntax score after adjusting confounders $(\beta=1.09,95 \%$ CI: $0.27,1.91, \mathrm{P}=0.0096$ ). By interaction and stratified analyses, the interactions were observed based on our specification including with the medication history of statins and angiotensin receptor blockers (ARBs) (P values for interaction $<0.05$ ).

Conclusion: In this study, we found a positive correlation between the HbA1c levels and the SYNTAX score among CAD individuals, and oral statins and ARBs medication could affect the correlation. Thus, HbAlc measurement could be used for the evaluation of the severity and complexity of coronary lesions among CAD patients.

Keywords: HbA1c, SYNTAX score, coronary artery disease, statins, ARBs

\section{Introduction}

Coronary Artery Disease (CAD) is the leading cause of death worldwide. Major efforts have been made to find novel useful non-invasive biomarkers for CAD. The biomarkers may be involved in the pathogenesis of CAD or the formation of risk factors, such as DNA methylation signatures affecting HLA-G gene were correlated with plaque load changes, which was predictive for disease severity. ${ }^{1}$ Glycated hemoglobin (HbA1c) is a product of the binding of hemoglobin and blood sugar in red blood cells in human blood. The HbAlc test usually reflects the patient's blood glucose control for nearly 8 to 12 weeks. There are lots of researches elaborating the effects of glycemic metabolism on CAD. ${ }^{2-10}$ These $^{-1}$ studies show that HbA1C were correlated positively with the Severity of CAD in different groups of people, such as older patients with diabetes mellitus, ${ }^{8}$ Type 2 Diabetes Mellitus, ${ }^{7}$ Non-Diabetic Subjects, ${ }^{4,6}$ Diabetic and Non-Diabetic Subjects, ${ }^{3,9}$ PCI Patients With HbA1c 5.7\% to $6.4^{10}$ and Non-diabetic Patients with Acute Coronary Syndrome ${ }^{5}$. However, previous studies regarding the relationship between HbA1c and SYNTAX score just adjusted some conventional risk factor, not adjusted conventional cardiovascular drugs. Patients with CAD need to take a large number of 
cardiovascular drugs for a long time, and these drugs have important impact on the outcome of CAD. SYNTAX scoring was proposed by The LANT European and American Guidelines as a tool to help clinicians determine the best revascularization method for patients with complex CAD. ${ }^{11}$ The higher the SYNTAX score, the more complex the lesion, and complex lesions often indicate undesirable consequences. It can effectively predict the occurrence of cardiogenic death and the incidence of major adverse cardiovascular and cerebrovascular events (MACCE). ${ }^{12}$ Therefore, this study was set out to investigate whether HbAlc is independently related to SYNTAX score in Chinese patients with CAD after adjusting conventional cardiovascular drugs, and subgroup analysis according to the history of Medications.

\section{Patients and Methods}

\section{Study Population}

We retrospectively reviewed $2990 \mathrm{CAD}$ patients aged 18 years and older who underwent their first coronary angiography (CAG) due to chest tightness and chest pain, to evaluate suspected CAD, at the Chinese Affiliated Hospital of Jining Medical College between July 2016 and July 2018. The clinical diagnosis and treatment process of each participant was completely in accordance with AHA/ACC guidelines. Inclusion criteria included (1) Stable angina; (2) Unstable angina; (3) ST-segment elevation myocardial infarction; (4) Non-ST segment elevation myocardial infarction. Patients with Type I diabetes, Stroke $(n=5)$, Severe liver and kidney dysfunction $(n=116)$, Severe heart failure $(n=27)$ and some patients $(n=2270)$ whose HbA1c levels were not available during the hospitalization were excluded. A total of 572 patients were included in the final analysis. Patients were divided into 4 groups (Interquartile) according to the following HbA1c levels: $4.00-5.50 \%, 5.60-6.30 \%, 6.40-7.40 \%, 7.50-18.00 \%$. This study was approved by the ethics committee of Affiliated Hospital of Jining Medical University. We confirm that all methods were performed in accordance with the relevant guidelines and regulations. Because of the nature of retrospective cohort study and the data are anonymous, the requirement for informed consent was therefore waived.

\section{Methods}

All data was obtained from the hospital database. General condition: The age, gender, systolic pressure (SBP), diastolic pressure (DBP), smoking, body mass index (BMI), the disease history of type 2 diabetes, Hypertension, Dyslipidemia, and previous medication history of Aspirin, Clopidogrel, Beta blockers, Statins, Angiotensin converting enzyme inhibitors (ACEIs), Angiotensin II receptor blockage (ARBs), Calcium antagonist (CCBs), Metformin, Insulin, Spironolactone, Anxiolytics was collected. Meanwhile, some routine examinations were recorded. Patients were fasted for $12 \mathrm{~h}$, and venous blood was collected in the morning. Total cholesterol (TC), triglycerides (TG), high-density lipoprotein (HDL-C), low-density lipoprotein (LDL-C), urea nitrogen, uric acid (UA), fasting blood glucose (FBG), alanine aminotransferase (ALT), creatinine $(\mathrm{Cr}), \mathrm{HbAlc}$ were detected. HbA1C levels were measured with the BioRadD-10 using ion-exchange high-performance liquid chromatography. All patients underwent coronary angiography by qualified interventional cardiologists. The CAD severity was quantified with the Syntax score. The SYNTAX score was calculated by a computer program. The algorithm contained 12 questions. The first three questions were coronary dominant type, number of lesions, and number of vascular segments of the lesion. The maximum number of lesions was 12, and each lesion was crowned with 1, 2, 3, and so on. Each lesion may have involved one or more segments. The integral of each lesion was calculated by the segment that is involved. The last 9 questions were the poor features of the lesion, and the scores for each lesion were derived based on the poor features. The score of each lesion was added to obtain a SYNTAX score. Coronary angiograms were analyzed by two experienced observers who were blinded to the identities and clinical information of the patients.

\section{Statistical Analysis}

The continuous variables are presented as the means \pm SDs. The categorical variables are presented as proportions (percentages). We used $\chi^{2}$ (categorical variables), One-Way ANOVA test (normal distribution), or KrusckalWhllis $H$-test (skewed distribution) to test for differences among differentHbAlc groups (Quartile). Univariate and multivariate 
linear regression were employed. We constructed four models: Non-adjusted model adjusted for: None; Model 1 adjusted for Gender, Age, BMI, Smoking, Cr, TG, HDL-C, LDL-C, TC, SBP, DBP; Model 2 adjusted for model 1 plus previous disease history of Diabetes, Hypertension, Hyperlipidemia; Model 3 adjusted for Model 2 plus previous medication history of Aspirin, Clopidogrel, Beta blockers, Statins, ACEIs, ARBs, CCBs, Metformin, Insulin, Spironolactone, Anxiolytics. We conducted the generalized additive model and smooth curve fitting to address the nonlinearity of HbA1c and SYNTAX score. If nonlinearity was detected, we first calculated the inflection point using recursive algorithm, and then constructed a two-piecewise linear regression on both sides of the inflection point. The interaction and stratified analyses were performed using stratified linear regression models. We did a sensitivity analysis to ensure the robustness of data analysis. To verify the results of $\mathrm{HbA} 1 \mathrm{c}$ as the continuous variable and observe the possibility of nonlinearity, HbA1c were converted into a categorical variable, and calculated the $\mathrm{P}$ for trend. All the analyses were performed with the statistical software packages R (http://www.R-project.org, The R Foundation) and Empower Stats (http://www.empowerstats.com, X\&Y Solutions, Inc, Boston, MA). P values less than 0.05 (two-sided) were considered statistically significant.

\section{Results}

\section{Baseline Characteristics of Selected Participants}

A total of 572 participants were selected for the final data analysis after screening by inclusion and exclusion criteria. Baseline characteristics of these selected participants are shown in Table 1 according to Quartile of HbA1c. In general, the average age of the 572 selected participants was $61 \pm 9$ years, and about $55 \%$ of them were male. The average level of $\mathrm{HbA} 1 \mathrm{c}$ of all the enrolled patients is $(6.81 \pm 1.73) \%$. No statistically significant differences were detected in SBP, DBP, TC, LDL-C, ALT, Smoking, the disease history of Hypertension, Hyperlipidemia, and the Medication history of Statins, ACEIs, ARBs, CCBs, Digoxin, Anxiolytics among different Glycated hemoglobin groups (all p values >0.05). Participants with the highest group of HbA1c (7.50-18.00\%) had the higher values in SBP, FBG, TG, TC, SYNTAX score, and consisted of significantly higher number of patients Smoking, with the disease history of Diabetes, Hyperlipidemia, and the Medication history of Aspirin, Clopidogrel, Beta blockers, Nitrates, Digoxin, Spironolactone, Metformin, and Insulin than those of the other groups. The opposite patterns were observed in the number of patients without the disease history of Diabetes, and the medication history of Aspirin, Clopidogrel, Beta blockers, Nitrates, and Insulin.

\section{Unvaried Analysis for SYNTAX Score}

We listed the results of univariate analyses in Table 2 by univariate linear regression, we found that BMI, SBP, TC, TG, ALT, Cr, UA, Gender, the disease history of Hyperlipidemia and the medication history of ACEIs, Statins, ARBs, Metformin were not associated with SYNTAX score. We also found that DBP $(-0.08,95 \% \mathrm{CI}:-0.15,-0.01 ; \mathrm{P}=0.02)$, HDL-C $(-5.89,95 \%$ CI: $-8.93,-2.84 ; \mathrm{P}=0.0002)$, and the medication history CCBS $(-3.05,95 \%$ CI: $-4.85,-1.25 ; \mathrm{P}=$ $0.0010)$, Anxiolytics $(-8.97,95 \% \mathrm{CI}:-13.50,-4.43 ; \mathrm{P}=0.0001)$ were negatively associated with SYNTAX score. In contrast, univariate analysis showed that Age (0.18, 95\% CI: 0.08, 0.28; P = 0.0003), LDL-C mmol/L (1.66, 95\% CI: 0.60, 2.72; P = 0.0023), FBG mmol/L (0.73, 95\% CI: 0.40, 1.06; P < 0.0001), GHbA1c\% (1.49, 95\% CI: 0.99, 2.00; P < $0.0001)$, Smoking $(2.74,95 \%$ CI: 0.96, 4.52; P < 0.0026), with the disease history of Diabetes $(3.22,95 \%$ CI: 1.40, 5.03; $\mathrm{P}<0.0006)$ and Hypertension (2.07, 95\% CI: $0.25,3.89 ; \mathrm{P}=0.0266)$, with the medication history of Aspirin $(9.74,95 \%$ CI: 4.44, 15.03; P = 0.0003), Clopidogrel (12.85, 95\% CI: 10.84, 14.85; P < 0.0001), Beta blockers (4.01, 95\% CI: 1.96, 6.06; $\mathrm{P}=0.0001)$, Nitrates (8.14, 95\% CI: 6.45, 9.82; P < 0.0001), Insulin (3.62, 95\% CI: 1.18, 6.06; P = 0.0037), Digoxin (13.57, 95\% CI: 8.50, 18.65; P < 0.0001), and Spironolactone (9.63, 95\% CI: 7.68, 11.57; P < 0.0001) were positively correlated with SYNTAX score.

\section{Results of Unadjusted and Adjusted Linear Regression}

In this study, we constructed four models to analyze the independent effects of HbA1c on SYNTAX score (univariate and multivariate linear regression). The effect sizes $(\beta)$ and $95 \%$ confidence intervals are listed in Table 3 . In the unadjusted 
Table I Baseline Characteristics of Participants

\begin{tabular}{|c|c|c|c|c|c|}
\hline GHbA Ic Quarter & QI $4.00-5.50 \%$ & Q2 5.60-6.30\% & Q3 6.40-7.40\% & Q4 7.50-18.00\% & P-value \\
\hline $\mathbf{N}$ & 130 & 148 & 143 & 151 & \\
\hline Age (Years) & $58.92 \pm 9.26$ & $61.76 \pm 8.72$ & $61.66 \pm 9.47$ & $61.41 \pm 8.98$ & 0.032 \\
\hline BMI $\left(\mathrm{kg} / \mathrm{m}^{2}\right)$ & $24.85 \pm 3.19$ & $26.18 \pm 3.26$ & $26.57 \pm 3.53$ & $26.30 \pm 3.49$ & 0.007 \\
\hline SBP (mmHg) & $132.38 \pm 18.46$ & $135.54 \pm 19.53$ & $136.92 \pm 22.46$ & $137.04 \pm 20.12$ & 0.199 \\
\hline DBP (mmHg) & $81.26 \pm 12.63$ & $80.08 \pm 12.98$ & $79.78 \pm 13.89$ & $80.14 \pm 12.53$ & 0.800 \\
\hline FBG (mmol/L) & $5.76 \pm 1.12$ & $6.55 \pm 1.88$ & $7.27 \pm 2.00$ & $10.06 \pm 3.26$ & $<0.001$ \\
\hline TG (mmol/L) & $1.41 \pm 0.80$ & $1.54 \pm 0.82$ & $1.74 \pm 0.92$ & $2.01 \pm 1.03$ & $<0.001$ \\
\hline TC (mmol/L) & $4.62 \pm 1.15$ & $4.57 \pm 1.01$ & $4.67 \pm 1.16$ & $4.68 \pm 1.18$ & 0.821 \\
\hline LDL-C (mmol/) & $2.67 \pm 0.81$ & $2.58 \pm 0.85$ & $2.71 \pm 0.89$ & $2.70 \pm 0.85$ & 0.576 \\
\hline HDL-C (mmol/L) & $1.21 \pm 0.32$ & $1.18 \pm 0.33$ & $1.10 \pm 0.25$ & $1.04 \pm 0.26$ & $<0.001$ \\
\hline $\mathrm{Cr}(\mu \mathrm{mol} / \mathrm{L})$ & $64.91 \pm 13.87$ & $60.77 \pm 13.18$ & $59.83 \pm 14.22$ & $58.32 \pm 14.16$ & 0.001 \\
\hline ALT (U/L) & $31.20 \pm 17.97$ & $29.72 \pm 15.69$ & $27.39 \pm 14.94$ & $27.87 \pm 16.76$ & 0.219 \\
\hline UA ( $\mu \mathrm{mol} / \mathrm{L})$ & $320.65 \pm 91.69$ & $300.6 I \pm 85.6 I$ & $293.13 \pm 87.87$ & $293.12 \pm 99.29$ & 0.054 \\
\hline SYNTAX score & $9.31 \pm 8.78$ & $12.46 \pm 10.80$ & $14.53 \pm 11.07$ & $17.10 \pm 11.09$ & $<0.001$ \\
\hline Male & $85(65.38)$ & 79 (53.38) & $69(48.25)$ & $80(52.98)$ & 0.034 \\
\hline Smoking & $68(52.31)$ & $64(43.24)$ & $54(37.76)$ & 7I (47.02) & 0.099 \\
\hline T2DM & $29(22.31)$ & $69(46.62)$ & II 15 (80.42) & I 44 (96.00) & $<0.001$ \\
\hline Hypertension & 74 (56.92) & $94(63.5 \mathrm{I})$ & $96(67.13)$ & 91 (60.67) & 0.351 \\
\hline Hyperlipidemia & $5(3.85)$ & $8(5.4 I)$ & $8(5.59)$ & II (7.33) & 0.655 \\
\hline Aspirin & $126(96.92)$ & I 40 (94.59) & I $39(97.20)$ & $147(100.00)$ & 0.048 \\
\hline Clopidogrel & 93 (7I.54) & II 6 (78.38) & II 18 (82.52) & 134 (91.16) & $<0.001$ \\
\hline Beta blockers & $86(66.15)$ & 112 (75.68) & II 4 (79.72) & $120(81.63)$ & 0.014 \\
\hline Statins & 110 (84.62) & I24 (83.78) & $133(93.01)$ & $|3|$ (89.12) & 0.064 \\
\hline ACEIS & $39(30.00)$ & $52(35.14)$ & $45(31.47)$ & $44(29.93)$ & 0.754 \\
\hline ARB & $18(13.85)$ & $15(10.14)$ & $18(12.59)$ & $25(17.01)$ & 0.374 \\
\hline CCBs & $47(36.15)$ & $57(38.5 I)$ & $64(44.76)$ & 51 (34.69) & 0.313 \\
\hline Nitrates & $62(47.69)$ & $89(60.14)$ & $93(65.03)$ & $100(68.03)$ & 0.003 \\
\hline Digoxin & $2(1.54)$ & $3(2.03)$ & $5(3.50)$ & $7(4.73)$ & 0.378 \\
\hline Spironolactone & $30(23.26)$ & 31 (20.95) & 31 (21.68) & $40(27.21)$ & 0.587 \\
\hline Anxiolytics & $4(3.08)$ & $8(5.4 I)$ & $5(3.50)$ & $5(3.40)$ & 0.730 \\
\hline Metformin & $3(2.31)$ & $6(4.05)$ & II (7.69) & $18(12.24)$ & 0.004 \\
\hline Insulin & $0(0.00)$ & $4(2.72)$ & $24(16.78)$ & $59(40.14)$ & $<0.001$ \\
\hline
\end{tabular}

Notes: Diabetes mellitus type 2, hypertension, hyperlipidemia represents the past disease history, drug represents a past medication history.

Abbreviations: GHbAlc, glycated hemoglobin Alc; BMI, body mass index; SBP, systolic blood pressure; DBP, diastolic blood pressure; FBG, fasting blood glucose; TG, triglycerides; TC, total cholesterol; LDL-C, low density lipoprotein cholesterol; HDL-C, high density lipoprotein cholesterol; Cr, creatinine; ALT, alanine aminotransferase; UA, uric acid; T2DM, diabetes mellitus type 2; ACEIS, angiotensin converting enzyme inhibitors; ARBs, angiotensin II receptor blockage; CCBS, calcium antagonist.

model, the model-based effect size can be explained as the difference in per mmol/L of HbAlc associated with Syntax score. For example, the effect size of 1.49 for SYNTAX score in unadjusted model means that a difference in per mmol/L of $\mathrm{HbA} 1 \mathrm{c}$ is associated with increased 1.49 difference in Syntax score $(95 \% \mathrm{CI}$ : 0.99-2.00, P $<0.0001)$. In the model 1 (adjusted for Gender, Age, BMI, Smoking, Cr, TG, HDL-C, LDL-C, TC, SBP, DBP), theHbA1c was increased per mmol/L, Syntax score increased by $1.17(95 \% \mathrm{CI}$ : 0.40-1.93, $\mathrm{P}=0.0031)$. In the model 2 (adjusted model 1 plus previous disease history of Diabetes, Hypertension, Hyperlipidemia) for each additional mmol/L of HbA1c, Syntax score increased by 1.14 (95\% CI: 0.24-2.04, P = 0.0142). In the model 3 (adjusted for Model 2 plus previous medication history of Aspirin, Clopidogrel, Beta blockers, Statins, ACEIs, ARBs, CCBs, Metformin, Insulin, Spironolactone, Anxiolytics), the HbA1c was increased per mmol/L, Syntax score increased by 1.09 (95\% CI: $0.27-1.91, \mathrm{P}=0.0096$ ). For the purpose of sensitivity analysis, we converted the HbAlc from continuous variable to categorical variable (Quartile of $\mathrm{HbAlc}$ ), the P for trend of $\mathrm{HbAlc}$ with categorical variables in fully adjusted model was consistent with the result when $\mathrm{HbAlc}$ is a continuous variable. Besides, we also found the trend of the effect size in different HbAlc groups was equidistant. 
Table 2 Univariate Analysis for SYNTAX Score

\begin{tabular}{|c|c|c|c|}
\hline Covariate & Statistics & $\beta(95 \% \mathrm{Cl})$ & P-value \\
\hline Age, year & $61.00 \pm 9.15$ & $0.18(0.08,0.28)$ & 0.0003 \\
\hline BMI, $\mathrm{kg} / \mathrm{m}^{2}$ & $25.96 \pm 3.42$ & $0.02(-0.33,0.37)$ & 0.8964 \\
\hline SBP, mmHg & $135.56 \pm 20.25$ & $-0.00(-0.05,0.04)$ & 0.9527 \\
\hline DBP, $\mathbf{m m H g}$ & $80.29 \pm 13.00$ & $-0.08(-0.15,-0.01)$ & 0.0200 \\
\hline $\mathrm{TC}, \mathrm{mmol} / \mathrm{L}$ & $4.63 \pm 1.12$ & $0.39(-0.42,1.19)$ & 0.3504 \\
\hline TG, $\mathrm{mmol} / \mathrm{L}$ & $1.68 \pm 0.92$ & $-0.03(-1.04,0.98)$ & 0.9493 \\
\hline LDL-C, mmol/L & $2.66 \pm 0.85$ & I.66 $(0.60,2.72)$ & 0.0023 \\
\hline HDL-C, $\mathrm{mmol} / \mathrm{L}$ & $1.13 \pm 0.30$ & $-5.89(-8.93,-2.84)$ & 0.0002 \\
\hline FBG, $\mathbf{m m o l} / \mathbf{L}$ & $7.45 \pm 2.75$ & $0.73(0.40,1.06)$ & $<0.0001$ \\
\hline ALT, U/L & $28.99 \pm 16.37$ & $0.03(-0.02,0.09)$ & 0.2596 \\
\hline $\mathrm{Cr}, \mu \mathrm{mol} / \mathrm{L}$ & $60.83 \pm 14.02$ & $0.03(-0.04,0.10)$ & 0.3605 \\
\hline UA, $\mu \mathrm{mol} / \mathrm{L}$ & $301.36 \pm 91.64$ & $-0.00(-0.01,0.01)$ & 0.7604 \\
\hline GHbAIc,\% & $6.81 \pm 1.73$ & $1.49(0.99,2.00)$ & $<0.0001$ \\
\hline Male & $313(54.72 \%)$ & $0.82(-0.97,2.61)$ & 0.3700 \\
\hline Smoking & 257 (44.93\%) & $2.74(0.96,4.52)$ & 0.0026 \\
\hline T2DM & 357 (62.52\%) & $3.22(1.40,5.03)$ & 0.0006 \\
\hline Hypertension & $355(62.17 \%)$ & $2.07(0.25,3.89)$ & 0.0266 \\
\hline Hyperlipidemia & $32(5.60 \%)$ & $-0.43(-4.29,3.43)$ & 0.8274 \\
\hline Aspirin & $552(97.18 \%)$ & $9.74(4.44,15.03)$ & 0.0003 \\
\hline Clopidogrel & $46 I$ (8I.16\%) & $12.85(10.84,14.85)$ & $<0.0001$ \\
\hline Beta blockers & $432(76.06 \%)$ & $4.01(1.96,6.06)$ & 0.0001 \\
\hline ACEIS & 180 (31.69\%) & $-0.87(-2.78,1.03)$ & 0.3681 \\
\hline Statins & $498(87.68 \%)$ & $-0.16(-2.85,2.54)$ & 0.9093 \\
\hline ARBs & $76(13.38 \%)$ & $-0.28(-2.89,2.32)$ & 0.8316 \\
\hline CCBS & $219(38.56 \%)$ & $-3.05(-4.85,-1.25)$ & 0.0010 \\
\hline Nitrates & 344 (60.56\%) & $8.14(6.45,9.82)$ & $<0.0001$ \\
\hline Metformin & 38 (6.69\%) & $-1.69(-5.24,1.85)$ & 0.3493 \\
\hline Insulin & 87 (I5.37\%) & $3.62(1.18,6.06)$ & 0.0037 \\
\hline Digoxin & 17 (2.99\%) & | $3.57(8.50,18.65)$ & $<0.0001$ \\
\hline Spironolactone & $132(23.28 \%)$ & $9.63(7.68,11.57)$ & $<0.0001$ \\
\hline Anxiolytics & $22(3.87 \%)$ & $-8.97(-13.50,-4.43)$ & 0.0001 \\
\hline
\end{tabular}

Abbreviations: $\mathrm{Cl}$, confidence interval; BMI, body mass index; SBP, systolic blood pressure; DBP, diastolic blood pressure; TC, total cholesterol; TG, triglycerides; LDL-C, low density lipoprotein cholesterol; HDL-C, high density lipoprotein cholesterol; FBG, fasting blood glucose; ALT, alanine aminotransferase; Cr, creatinine; UA, uric acid; GHbAlc, glycated hemoglobin Alc; T2DM, diabetes mellitus type 2; ACEls, angiotensin converting enzyme inhibitors; ARBs, angiotensin II receptor blockage; CCs, calcium channel antagonists.

\section{The Results of Nonlinearity of HbAIc and SYNTAX Score}

In the present study, we analyzed the non-linear relationship between HbA1c and SYNTAX score. Smooth curve and the result of Generalized additive model showed that the relationship between HbAlc and SYNTAX score was non-linear after unadjusted for Age, Gender, BMI, TG, TC, LDL-C, HDL-C. Because the P (0.043) for log likelihood ratio test was less than 0.05 , therefore, we chose two-piecewise linear regression for fitting the association between HbA1c and SYNTAX score because it can accurately represent the relationship. The non-linear relationship between HbAlc and SYNTAX score are listed in Figure 1. By two-piece wise linear regression and recursive algorithm, we calculated the inflection point was 5.9mmol/L. On the left side of inflection point, the effect size and $95 \% \mathrm{CI}$ were $4.34(1.10,7.57),(\mathrm{P}=0.009)$ respectively. On the right side of inflection point, the effect size and $95 \% \mathrm{CI}$ were $0.64(-0.18,1.47)$, and $(\mathrm{P}=0.1293)$, respectively (Table 4).

\section{The Interaction and Stratified Analyses of the Affect and Association Between HbAlc and SYNTAX Score}

We examined whether Diabetes, Hypertension, Hyperlipidemia status, and previous medication history affected the association between HbA1c and SYNTAX score. After adjusting for Age, BMI, SBP, DBP, TC, TG, LDL-C, HDL-C, 
Table 3 Relationship Between GHbAlc and SYNTAX Score in Different Models

\begin{tabular}{|c|c|c|c|c|}
\hline \multirow[t]{2}{*}{ Exposure } & Non-Adjusted & Model I & Model 2 & Model 3 \\
\hline & $\beta(95 \% \mathrm{Cl}) \mathrm{P}$-value & $\beta(95 \% \mathrm{Cl})$ P-value & $\beta(95 \% \mathrm{Cl})$ P-value & B (95\% Cl) P-value \\
\hline $\begin{array}{l}\text { GHbAlc (\%) } \\
\text { GHbAlc quartile }\end{array}$ & I.49 $(0.99,2.00)<0.000$ I & $1.17(0.40,1.93) 0.0031$ & I.I $4(0.24,2.04) 0.0142$ & $1.09(0.27,1.91) 0.0096$ \\
\hline QI & Reference & Reference & Reference & Reference \\
\hline Q2 & $3.15(0.67,5.63) 0.0131$ & $3.27(-0.06,6.59) 0.0553$ & $3.33(-0.03,6.69) 0.0531$ & $1.48(-1.44,4.40) 0.3217$ \\
\hline Q3 & $5.22(2.72,7.72)<0.0001$ & $4.22(0.74,7.70) 0.0182$ & $4.21(0.27,8.15) 0.0372$ & $2.97(-0.16,6.10) 0.0645$ \\
\hline Q4 & $7.79(5.32,10.26)<0.0001$ & $5.70(2.09,9.30) 0.0022$ & $5.55(1.28,9.82) 0.0114$ & $4.20(0.7 I, 7.69) 0.0190$ \\
\hline$P$ for trend & $<0.0001$ & 0.0022 & 0.0130 & 0.0132 \\
\hline
\end{tabular}

Notes: Non-adjusted model adjusted for: none; model I adjusted for gender, age, BMI, smoking, Cr, TG, HDL-C, LDL-C, TC, SBP, DBP; model 2 adjusted for model I plus previous disease history of T2DM, hypertension, hyperlipidemia; model 3 adjusted for model 2 plus previous medication history of aspirin, clopidogrel, beta blockers, statins, ACEIS, ARBs, CCBs, metformin, insulin, spironolactone, anxiolytics.

Abbreviations: $\mathrm{Cl}$, confidence interval; BMI, body mass index; $\mathrm{Cr}$, creatinine; TG, triglycerides; HDL-C, high density lipoprotein cholesterol; LDL-C, low density lipoprotein cholesterol; TC, total cholesterol; SBP, systolic blood pressure; DBP, diastolic blood pressure; T2DM, diabetes mellitus type 2; ACEIS, angiotensin converting enzyme inhibitors; ARBs, angiotensin II receptor blockage; CCBs, calcium channel antagonist.

UA, Cr, ALT except the subgroup variable, as shown in Figure 2, we noted that only a small number of interactions were observed based on our a priori specification including: the medication history of Statins and ARBs (P values for interaction $<0.05$ ). In this study, the stronger association of HbAlc and SYNTAX score were detected in no Diabetes, Hypertension, Hyperlipidemia status, and no previous medication history of Statins, ARBs, CCBs, Metformin, Beta

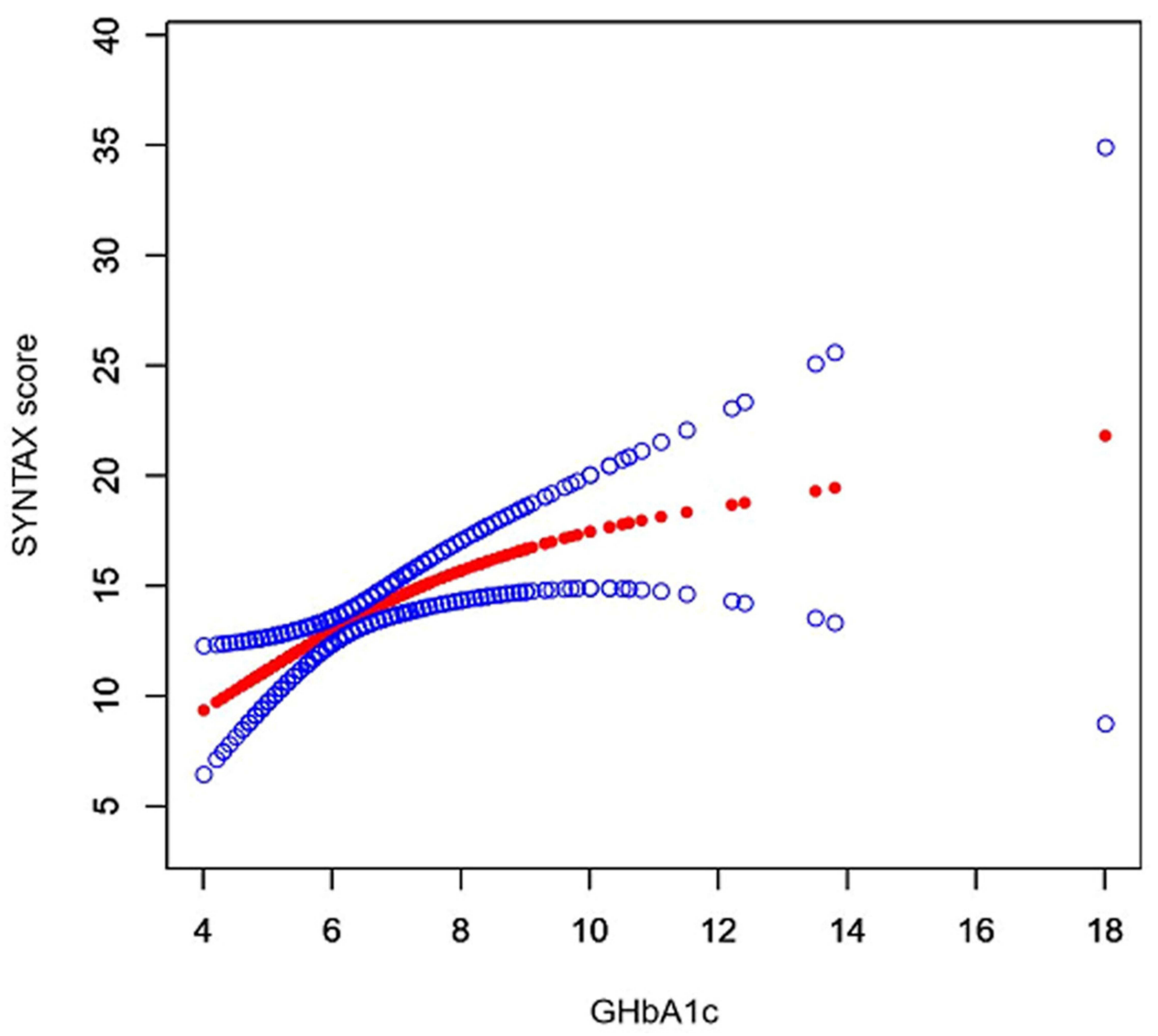

Figure I Association between GHbAlc and SYNTAX score in coronary heart disease patients. A threshold, nonlinear association between GHbAIc and SYNTAX score was found $(P=0.0052)$ in a generalized additive model $(G A M)$. Solid red line represents the smooth curve fit between variables. Blue bands represent the $95 \%$ of confidence interval from the fit. All adjusted for age, gender, BMI, TG, TC, LDL-C, HDL-C.

Abbreviations: BMI, body mass index; TG, triglycerides; HDL-C, high density lipoprotein cholesterol; LDL-C, low density lipoprotein cholesterol; TC, total cholesterol. 
Table 4 Threshold Effect Analysis of GHbAIc and SYNTAX Score Using Piece-Wise Linear Regression

\begin{tabular}{|l|l|l|l|}
\hline Inflection Point of & Effect Size $(\boldsymbol{\beta})$ & $\mathbf{9 5 \%} \mathbf{~ C l}$ & P value \\
\hline 5.9 & & & \\
$<5.9$ & 4.34 & $1.10,7.57$ & 0.0090 \\
$>5.9$ & 0.64 & $-0.18,1.47$ & 0.1293 \\
\hline
\end{tabular}

Notes: Effect: SYNTAX score, cause: GHbAIc, adjusted: age, gender, BMI, TG, TC, LDL-C, HDL-C.

Abbreviations: BMI, body mass index; TG, triglycerides; HDL-C, high density lipoprotein cholesterol; LDLA -C, low density lipoprotein cholesterol; TC, total cholesterol.

blockers, Insulin, Digoxin, Spironolactone, Anxiolytics $(<0.05 \mathrm{P})$. In contrast, the weaker association were detected between men and female with disease history of Diabetes, Hypertension, and with previous medication history of Statins, ARBs, CCBs, Beta blockers, Insulin, Spironolactone, with and without the medication history of Clopidogrel, Nitrates, and ACEIs.

\section{Discussion}

In this study, a retrospective cohort study was performed to address the relationship between HbA1c and the SYNTAX score, we found that HbAlc was positively associated with SYNTAX score after adjusting a wide range of known cardiovascular risk factors and medication history. We also found that there were different trends on the left and right sides of the inflection point [left 5.9 (95\% CI: 1.10-7.57); right 0.64 (95\% CI: $-0.18-1.47)$ ] after adjusting a range of known cardiovascular risk factors and medication history. The results showed that the history of medication had some influence on the relationship of HbA1c and SYNTAX score. We performed an interaction and stratified analyses to the confounders, which helped us to better understand the trend of HbA1c and SYNTAX score in different populations. The results of interaction and stratified analyses found that the medication history of Statins and ARBs had a significant impact on the relationship of HbA1c and SYNTAX score. With increasing HbA1c levels, a marked increase in SYNTAX score was noted in the patients without a history of taking Statins [8.13 (95\% CI: 3.15, 13.11)]. However, Patients with a history of taking Statins tended to less increase in SYNTAX score [0.61 (95\% CI: $-0.19,1.42)]$. Patients without a history of taking ARBs tended to increase in SYNTAX score [1.30 (95\% CI: $0.42,2.18)]$, but patients with a history of taking ARBs tended to decrease in SYNTAX score [-0.86 (95\% CI: $-3.14,1.42)]$.

Epidemiologic studies show that $\mathrm{HbAlc}$ is a cardiovascular risk factor in all kinds of individuals. ${ }^{3-10}$ Our findings are in accordance with prior studies that demonstrated that $\mathrm{HbAlc}$ levels are independently associated with SYNTAX score which represents the degree of coronary atherosclerosis in non-diabetic and diabetic individuals. This association is graded, continuous, and independent of conventional major cardiovascular risk factors and history of taking cardiovascular related drugs. HbA1c could be used as an independent predictor of CAD and its severity even in non-diabetic and diabetic individuals. Since HbA1c reflects the blood glucose control situation of the last 2-3 months, with less biological variability and greater stability, can be assessed in the non-fasting state, it is a more convenient test than fasting blood glucose. Some studies ${ }^{2,4}$ have shown that fasting blood glucose is not related to CAD, therefore we did not detect fasting blood glucose. The population we collected is patients with acute coronary syndrome and undergoing coronary angiography, including diabetic and non-diabetic patients. All the above studies were only adjusted for traditional cardiovascular risk factors. We adjusted the history of cardiovascular medication and performed a subgroup analysis to highlight the important effect of medication on the correlation between HbA1c and SYNTAX score. We found that oral Statins and ARBs drugs in patients with coronary heart disease can significantly improve the increase in SYNTAX score caused by increased HbA1c.

Statins are widely used to control dyslipidemia. Some studies ${ }^{1,13}$ have shown that long-term use of Statins is related to diabetes mellitus (DM) and impaired fasting Blood glucose (IFG), more marked in people with prediabetes, metabolic syndrome, and intensive statins therapy. However, meta-analyses of randomized clinical trials data have revealed the benefits of Statins in preventing cardiovascular disease (CVD) which events far outweigh the potential risk from elevation in plasma glucose. ${ }^{14,15}$ In our study, patients with diabetes and non-diabetic patients were selected, we found that the use of Statins can improve the relationship between HbA1c and SYNTAX score, which validated the conclusion 


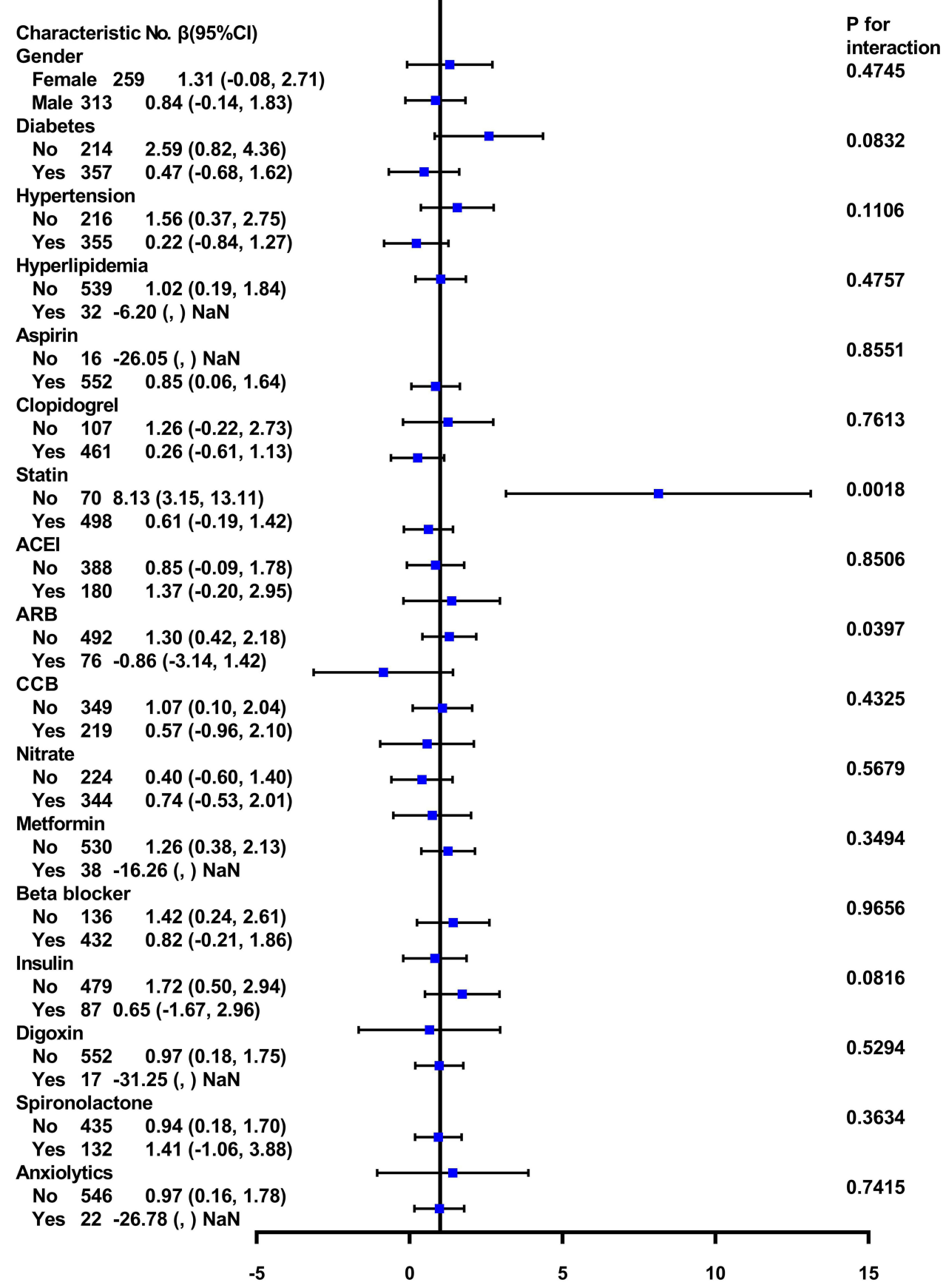

Figure 2 The interacting and stratified analyses of affecting the association between HbAlc and SYNTAX score. Adjusted for season of age, BMI, SBP, DBP, TC, TG, LDL-C, HDL-C, UA, Cr, ALT except the variable. Small number of interactions were observed based including: the medication history of statins and ARBs (P values for interaction $<0.05)$. In this study, the stronger association of HbAlc and SYNTAX score were detected in no diabetes, hypertension, hyperlipidemia status, and no previous medication history of statins, ARBs, CCBs, metformin, beta blockers, insulin, digoxin, spironolactone, anxiolytics $(<0.05 \mathrm{P})$. In contrast, the weaker association was detected between men and female with disease history of diabetes, hypertension, and with previous medication history of statins, ARBs, CCBS, beta blockers, insulin, spironolactone, with and without the medication history of clopidogrel, nitrates, ACEls.(,) $\mathrm{NaN}$ : the model failed because of the small sample size.

Abbreviations: $\mathrm{Cl}$, confidence interval; ACEIS, angiotensin converting enzyme inhibitors; ARBs, angiotensin II receptor blockage; CCBS, calcium antagonist; BMI, body mass index; $\mathrm{Cr}$, creatinine; TG, triglycerides; HDL-C, high density lipoprotein cholesterol; LDL-C, low density lipoprotein cholesterol; TC, total cholesterol; SBP, systolic blood pressure; DBP, diastolic blood pressure.

that the efficacy of Statins in preventing CVD events far outweighs the potential risk of incidental plasma glucose elevation.

Prolonged exposure to hyperglycemia is now recognized as a major factor in the pathogenesis of diabetic complications, including atherosclerosis. Hyperglycemia induces a large number of alterations at the cellular level of vascular 
tissue that potentially accelerates the atherosclerotic process. Animal and human studies have provided the major mechanisms: ${ }^{16}$ (1) The hyperglycemia strengthens the process of overproduction of superoxide by the mitochondrial electron-transport chain, which increases reactive oxygen species (ROS) through several pathways. ${ }^{17-21}$ (2) Hyperglycemia promotes inflammation through the induction of cytokine secretion by several cell types including monocytes and adipocytes. ${ }^{21-24}$ The ROS and inflammation are the main factors which accelerates atherosclerosis and leads to increased risk of myocardial infarction, stroke. We found that oral Statins and ARBs drugs in patients with coronary heart disease can significantly improve the increase in SYNTAX score caused by increased HbAlc, which shows that Statins and ARBs drugs can improve Hyperglycemia-induced atherosclerosis. The mechanism of Statins improving vascular damage by hyperglycemia is not completely understood, but the prevailing theories include: improve endothelial dysfunction, ${ }^{25}$ anti-inflammatory, ${ }^{26}$ anti- ROS, ${ }^{27}$ anti-platelet activation, ${ }^{28}$ reduce vascular smooth muscle proliferation, ${ }^{29}$ anti-arrhythmia, etc. The mechanism of ARBs improving vascular damage by hyperglycemia may include: ARBs treatment may play an antioxidant role, ${ }^{30}$ improve cardiac mitochondrial activity, ${ }^{31}$ mitigate hyperglycemia-induced vascular inflammation, ${ }^{32}$ anti-fibrosis, ${ }^{33}$ and so on.

This study strengthens the perception that the simple laboratory measurement of HbAlc in CAD patients with diabetic and non-diabetic will enable recognition of high-risk population, give Statins or ARBs drugs as much as possible that would benefit from aggressive intervention during hospitalization and closer follow-up. We demonstrate a continuous relation of $\mathrm{HbA1c}$ levels to $\mathrm{CAD}$ severity similarly to cholesterol and inflammatory biomarkers. This risk becomes evident in the study at anHbA1clevel of below $5.9 \%$ (pre-diabetes), which showed pre-diabetics should strictly control blood sugar, a slight increase in HbAlc can lead to serious progression of coronary heart disease. This risk reduced at an HbA1clevel of above $5.9 \%$, it may be due to the application of cardiovascular-related drugs, such as Statins, ARBs. Prospective studies linking HbAlc levels to CAD severity have been inconsistent and limited. Some studies ${ }^{34}$ have shown in non-diabetic subjects the optimal cut-off of $\mathrm{HbAlc}$ for the diagnosis of $\mathrm{CAD}$ in 5.6\%. Meanwhile, the outweigh the potential risk of incidental plasma glucose benefits of Statins in preventing CVD events far elevation. ARBs not ACEIS drugs improve the correlation between HbAlclevel and CAD severity. Future larger studies will be needed to verify our results to guide clinicians to patients at increased risk and advised about the patients at increased risk and advise them to consider aggressive coronary risk factor modification and management.

\section{Limitations}

Our study has several limitations. Due to the missed test of $\mathrm{HbA}_{1} \mathrm{c}$, the final sample size was small. Our research subjects are Chinese patients with CAD and it might not apply to other populations. Because we exclude Type I diabetes, Stroke, severe liver and kidney dysfunction, severe heart failure, therefore, the findings of this study cannot be used for these people. Since patients underwent one-time measurement of HbAlc and the cross-sectional study design, it just represented that the blood glucose levels in the last 3 months are significantly associated with the SYNTAX scores, and cannot determine the causal relationship.

\section{Conclusions}

In conclusion, the HbAlc is significantly associated with the SYNTAX scores which reflect the severity and complexity of coronary lesions in CAD patients with and without diabetes. This association is graded, continuous, even after adjusting the coronary atherosclerotic risk factors and conventional cardiovascular drugs. Thus, HbA1c may be a useful indicator of CAD patients with and without diabetes similar to cholesterol and inflammatory biomarkers. This risk becomes evident at an HbAlc level of below $5.9 \%$ (prediabetes). However, Statins and ARBs drugs can improve the correlation between HbA1c level and CAD severity. Controlling the HbAlc level and taking Statins and ARBs drugs in CAD patients may slow the progression of coronary atherosclerosis. Future larger studies will be needed to verify our results to guide clinicians about the patients at increased risk and advise them consider aggressive coronary risk factor modification and management. 


\section{Abbreviations}

HbA1c, Glycated hemoglobin A1c; CAD, Coronary Artery Disease; CAG, Coronary angiography; SBP, Systolic pressure; DBP, Diastolic pressure; BMI, Body mass index; ACEIs, Angiotensin converting enzyme inhibitors; ARBs, Angiotensin II receptor blockage; CCBS, Calcium antagonist; TC, Total cholesterol; TG, Triglycerides; HDL-C, Highdensity lipoprotein; LDL-C, Low-density lipoprotein; UA, Uric acid; UN, Urea nitrogen; FBG, Fasting blood glucose; ALT, Alanine aminotransferase; Cr, Creatinine; DM, Diabetes mellitus; IFG, Impaired fasting glucose; PKC, Protein Kinase C.

\section{Data Sharing Statement}

The data that support the findings of this study are available from the corresponding author upon reasonable request.

\section{Ethics Approval and Consent to Participate}

Participants informed consent is not required in this study because of the nature of retrospective cohort study. The study was approved by the ethics committee of Affiliated Hospital of Jining Medical University. The data are anonymous, and the requirement for informed consent was therefore waived.

\section{Acknowledgment}

The authors thank all the staff members in our institution.

\section{Author Contributions}

All authors made a significant contribution to the work reported, whether that is in the conception, study design, execution, acquisition of data, analysis and interpretation, or in all these areas; took part in drafting, revising or critically reviewing the article; gave final approval of the version to be published; have agreed on the journal to which the article has been submitted; and agree to be accountable for all aspects of the work.

\section{Funding}

This work was supported by Provincial Natural Science Foundation (ZR2017LH001), National Natural Science Foundation Youth Fund (81700230), Shandong Province Higher Educational Science and Technology Program (J16LL52), the PhD Research Startup Foundation of the Affiliated Hospital of Jining Medical University (2017-BS002), Jining Science and Technology Development Project, China ([2016]56-33, [2015]57-17).

\section{Disclosure}

The authors report no potential conflicts of interest relevant to this study.

\section{References}

1. Schiano C, Benincasa G, Infante T, et al. Integrated analysis of DNA methylation profile of HLA-G gene and imaging in coronary heart disease: Pilot study. PLoS One. 2020;15(8):e0236951. doi:10.1371/journal.pone.0236951

2. Arbel Y, Zlotnik M, Halkin A, et al. Admission glucose, fasting glucose, HbA1c levels and the SYNTAX score in non-diabetic patients undergoing coronary angiography. Clin Res Cardiol. 2014;103(3):223-227. doi:10.1007/s00392-013-0641-7

3. Basman C, Fishman SL, Avtanski D, et al. Glycosylated hemoglobin, but not advanced glycation end products, predicts severity of coronary artery disease in patients with or without diabetes. Metab Open. 2020;7:100050. doi:10.1016/j.metop.2020.100050

4. Garg N, Moorthy N, Kapoor A, et al. Hemoglobin A(1c) in nondiabetic patients: an independent predictor of coronary artery disease and its severity. Mayo Clin Proc. 2014;89(7):908-916. doi:10.1016/j.mayocp.2014.03.017

5. Habib S, Ullah SZ, Saghir T, et al. The association between hemoglobin A1c and the severity of coronary artery disease in non-diabetic patients with acute coronary syndrome. Cureus. 2020;12(1):e6631. doi:10.7759/cureus.6631

6. Ikeda N, Iijima R, Hara H, Moroi M, Nakamura M, Sugi K. Glycated hemoglobin is associated with the complexity of coronary artery disease, even in non-diabetic adults. $J$ Atheroscler Thromb. 2012;19(12):1066-1072. doi:10.5551/jat.13722

7. Karakoyun S, Gökdeniz T, Gürsoy MO, et al. Increased glycated hemoglobin level is associated with SYNTAX score II in patients with type 2 diabetes mellitus. Angiology. 2016;67(4):384-390. doi:10.1177/0003319715591752

8. Ma J, Wang X, Wang Y, Zhao Y, Gao M, Li X. The relationship between glycated hemoglobin and complexity of coronary artery lesions among older patients with diabetes mellitus. PLoS One. 2014;9(3):e91972. doi:10.1371/journal.pone.0091972 
9. Ul-Haque I, Ud Deen Z, Shafique S, et al. The role of glycated hemoglobin A1c in determining the severity of coronary artery disease in diabetic and non-diabetic subjects in Karachi. Cureus. 2019;11(6):e4982. doi:10.7759/cureus.4982

10. Yang J, Zhou Y, Zhang T, et al. Fasting blood glucose and $\mathrm{HbA}(1 \mathrm{c})$ correlate with severity of coronary artery disease in elective PCI patients with $\mathrm{HbA}(1 \mathrm{c}) 5.7 \%$ to 6.4. Angiology. 2020;71(2):167-174. doi:10.1177/0003319719887655

11. Ong AT, Serruys PW, Mohr FW, et al. The SYNergy between percutaneous coronary intervention with TAXus and cardiac surgery (SYNTAX) study: design, rationale, and run-in phase. Am Heart J. 2006;151(6):1194-1204. doi:10.1016/j.ahj.2005.07.017

12. Yang CH, Hsieh MJ, Chen CC, et al. SYNTAX score: an independent predictor of long-term cardiac mortality in patients with acute ST-elevation myocardial infarction. Coron Artery Dis. 2012;23(7):445-449. doi:10.1097/MCA.0b013e3283587835

13. Carter AA, Gomes T, Camacho X, Juurlink DN, Shah BR, Mamdani MM. Risk of incident diabetes among patients treated with statins: population based study. BMJ. 2013;346(may23 4):f2610. doi:10.1136/bmj.f2610

14. Açar B, Ozeke O, Karakurt M, et al. Association of prediabetes with higher coronary atherosclerotic burden among patients with first diagnosed acute coronary syndrome. Angiology. 2019;70(2):174-180. doi:10.1177/0003319718772420

15. Hammad MA, Abdo MS, Mashaly AM, et al. The statins effects on HbAlc control among diabetic patients: an umbrella review of systematic reviews and meta-analyses of observational studies and clinical trials. Diabetes Metab Syndr. 2019;13(4):2557-2564. doi:10.1016/j. dsx.2019.07.005

16. Aronson D. Hyperglycemia and the pathobiology of diabetic complications. Adv Cardiol. 2008;45:1-16. doi:10.1159/000115118

17. Brownlee M. Biochemistry and molecular cell biology of diabetic complications. Nature. 2001;414(6865):813-820. doi:10.1038/414813a

18. Akimoto Y, Hart GW, Hirano H, Kawakami H. O-GlcNAc modification of nucleocytoplasmic proteins and diabetes. Med Mol Morphol. 2005;38 (2):84-91. doi:10.1007/s00795-004-0264-1

19. Brownlee M. The pathobiology of diabetic complications: a unifying mechanism. Diabetes. 2005;54(6):1615-1625. doi:10.2337/diabetes.54.6.1615

20. Martinez MR, Dias TB, Natov PS, Zachara NE. Stress-induced O-GlcNAcylation: an adaptive process of injured cells. Biochem Soc Trans. 2017;45 (1):237-249. doi:10.1042/bst20160153

21. Wang JS, Huang Y, Zhang S, et al. A protective role of paeoniflorin in fluctuant hyperglycemia-induced vascular endothelial injuries through antioxidative and anti-inflammatory effects and reduction of PKCß1. Oxid Med Cell Longev. 2019;2019:5647219. doi:10.1155/2019/5647219

22. Murugan DD, Md Zain Z, Choy KW, et al. Edible bird's nest protects against hyperglycemia-induced oxidative stress and endothelial dysfunction. Front Pharmacol. 2019;10:1624. doi:10.3389/fphar.2019.01624

23. Peiró C, Romacho T, Azcutia V, et al. Inflammation, glucose, and vascular cell damage: the role of the pentose phosphate pathway. Cardiovasc Diabetol. 2016;15:82. doi:10.1186/s12933-016-0397-2

24. Dymkowska D, Drabarek B, Podszywałow-Bartnicka P, Szczepanowska J, Zabłocki K. Hyperglycaemia modifies energy metabolism and reactive oxygen species formation in endothelial cells in vitro. Arch Biochem Biophys. 2014;542:7-13. doi:10.1016/j.abb.2013.11.008

25. Leenders GJ, Smeets MB, van den Boomen M, et al. Statins promote cardiac infarct healing by modulating endothelial barrier function revealed by contrast-enhanced magnetic resonance imaging. Arterioscler Thromb Vasc Biol. 2018;38(1):186-194. doi:10.1161/atvbaha.117.310339

26. Kouhpeikar H, Delbari Z, Sathyapalan T, Simental-Mendía LE, Jamialahmadi T, Sahebkar A. The effect of statins through mast cells in the pathophysiology of atherosclerosis: a review. Curr Atheroscler Rep. 2020;22(5):19. doi:10.1007/s11883-020-00837-9

27. Nabi R, Alvi SS, Saeed M, Ahmad S, Khan MS. Glycation and HMG-CoA reductase inhibitors: implication in diabetes and associated complications. Curr Diabetes Rev. 2019;15(3):213-223. doi:10.2174/1573399814666180924113442

28. Lee YM, Chen WF, Chou DS, et al. Cyclic nucleotides and mitogen-activated protein kinases: regulation of simvastatin in platelet activation. $J$ Biomed Sci. 2010;17(1):45. doi:10.1186/1423-0127-17-45

29. Sanyour HJ, Li N, Rickel AP, et al. Statin-mediated cholesterol depletion exerts coordinated effects on the alterations in rat vascular smooth muscle cell biomechanics and migration. J Physiol. 2020;598(8):1505-1522. doi:10.1113/jp279528

30. Rodriguez R, Minas JN, Vazquez-Medina JP, et al. Chronic AT1 blockade improves glucose homeostasis in obese OLETF rats. $J$ Endocrinol. 2018;237(3):271-284. doi:10.1530/joe-17-0678

31. Thorwald M, Rodriguez R, Lee A, et al. Angiotensin receptor blockade improves cardiac mitochondrial activity in response to an acute glucose load in obese insulin resistant rats. Redox Biol. 2018;14:371-378. doi:10.1016/j.redox.2017.10.005

32. Song KH, Bae SJ, Chang J, et al. Telmisartan mitigates hyperglycemia-induced vascular inflammation by increasing GSK3 $\beta$-Ser(9) phosphorylation in endothelial cells and mouse aortas. Biochem Biophys Res Commun. 2017;491(4):903-911. doi:10.1016/j.bbrc.2017.07.134

33. Karihaloo A. Anti-fibrosis therapy and diabetic nephropathy. Curr Diab Rep. 2012;12(4):414-422. doi:10.1007/s11892-012-0290-7

34. Ashraf H, Boroumand MA, Amirzadegan A, Talesh SA, Davoodi G. Hemoglobin A1C in non-diabetic patients: an independent predictor of coronary artery disease and its severity. Diabetes Res Clin Pract. 2013;102(3):225-232. doi:10.1016/j.diabres.2013.10.011

International Journal of General Medicine

Dovepress

\section{Publish your work in this journal}

The International Journal of General Medicine is an international, peer-reviewed open-access journal that focuses on general and internal medicine, pathogenesis, epidemiology, diagnosis, monitoring and treatment protocols. The journal is characterized by the rapid reporting of reviews, original research and clinical studies across all disease areas. The manuscript management system is completely online and includes a very quick and fair peer-review system, which is all easy to use. Visit http://www.dovepress.com/testimonials.php to read real quotes from published authors.

Submit your manuscript here: https://www.dovepress.com/international-journal-of-general-medicine-journal 